\title{
Imaging of musculoskeletal lymphoma
}

\author{
Chee Yeong Lim, Keh Oon Ong \\ Department of Diagnostic Radiology, Singapore General Hospital, Block 2 Level 1 Outram Road, Singapore 169608 \\ Corresponding address: Dr Lim Chee Yeong, Department of Diagnostic Radiology Singapore General Hospital, Block 2 \\ Level 1 Outram Road Singapore 169608. \\ Email: drcylimegmail.com
}

Date accepted for publication 8 August 2013

\begin{abstract}
Lymphoma of the musculoskeletal system involving the bone, muscle or skin is commonly due to secondary involvement from disseminated disease but can occasionally present as primary extranodal disease. Although radiological features are traditionally known to be non-specific, recognition of certain characteristics using summation of imaging modalities as well as knowledge of clinical features can help in making the diagnosis. Imaging also plays an integral role in treatment response assessments, especially via positron emission tomography/computed tomography functional imaging.
\end{abstract}

Keywords: Lymphoma; bone; muscle; skin.

\section{Introduction}

Tumours of haematopoietic and lymphoid tissues are heterogeneous disease entities that are challenging to classify ${ }^{[1]}$. Malignant lymphomas, which include Hodgkin disease and non-Hodgkin lymphoma (NHL), are discussed in this pictorial review because of the established role of imaging in the diagnosis, staging and treatment response assessment ${ }^{[2]}$.

Lymphoma accounts for $4-6 \%$ of all cancers in the Singapore Cancer Registry in 2007-2011, similar to statistics found in the United States over the same period $^{[3,4]}$. Lymphoma occurs infrequently within bone, muscle or skin; this may represent secondary haematologic disease involvement or rarely a primary extranodal site of disease ${ }^{[5,6]}$. This review discusses the appearance of musculoskeletal lymphoma across various imaging modalities, with a brief discussion on post-treatment imaging.

\section{Bone disease}

Malignant lymphoma of bone accounts for $7 \%$ of all bone malignancies and $5 \%$ of extranodal lymphomas ${ }^{[7]}$. This is traditionally classified into four subtypes: primary bone lymphoma (PBL); multifocal PBL; disseminated lymphoma with secondary osseous involvement either within 6 months of lymphoma diagnosis via soft tissue/ nodal disease; or more than 6 months after diagnosis ${ }^{[5]}$.

PBL is defined as lymphoma within a single bone with or without regional nodal metastasis and the absence of distal lesions within 6 months after diagnosis ${ }^{[8]}$. Multifocal PBL is considered a less common subtype of PBL in which there is lymphomatous disease in two or more osseous sites but no distal or nodal metastasis within 6 months of diagnosis. The prognosis is poorer than with PBL but better than disseminated disease with secondary osseous involvement ${ }^{[5]}$.

Osseous lymphoma is usually due to NHL, with diffuse large cell lymphoma the most common cell type ${ }^{[5,9]}$. Clinical presentations include bone pain, B-symptoms and soft tissue swelling, pathologic fracture and cord compression $^{[10-12]}$. PBL shows a male preponderance, is rare in the first decade and occurs at a mean age of 42 years $^{[13]}$.

PBL is commonly found in the appendicular skeleton, typically affecting the metadiaphysis of the femur, tibia and humerus. Localized disease within the epiphysis, metaphysis or diaphysis of long bones is also noted. Axial flat bones such as the pelvis, scapula, ribs and vertebral bodies are infrequently encountered. Tumour may cross to adjacent bone by invading joint or vertebral spaces, a sign that when present helps to narrow the differential diagnosis (including infection, chordoma, 
chondrosarcoma and metastasis $)^{[13,14]}$. Multifocal PBLs can have both axial and appendicular lesions, commonly in the distal femur, proximal tibia and spine ${ }^{[15]}$. Secondary osseous lymphoma, on the contrary, preferentially affects the axial skeleton such as the spine, pelvis, skull, ribs and facial bones ${ }^{[9]}$.

Radiographic features of osseous lymphoma are variable. Some common features are described, but no pathognomonic sign is reliable to identify or differentiate osseous lymphoma. Radiological appearance can be normal, lytic, mixed lytic/sclerotic or sclerotic.

(a)

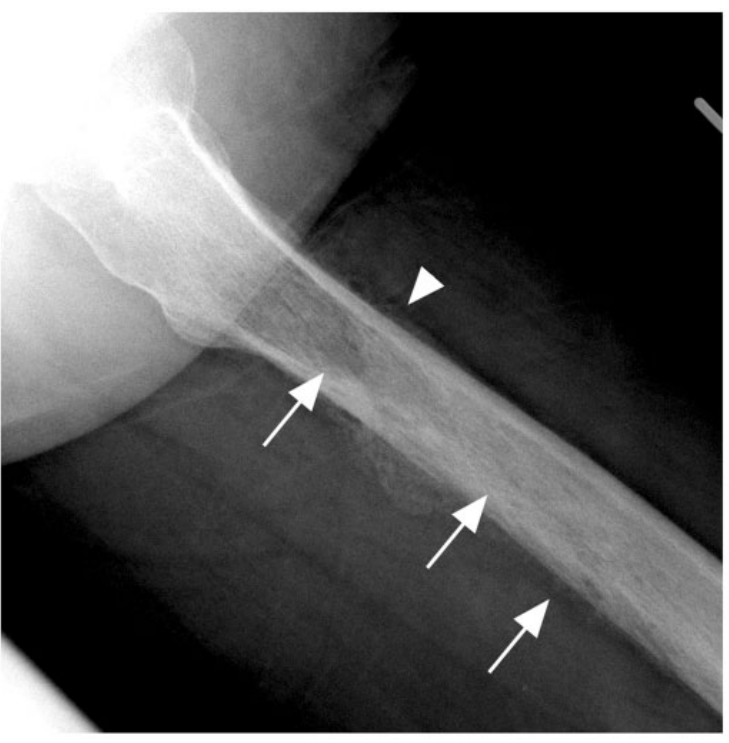

(c)

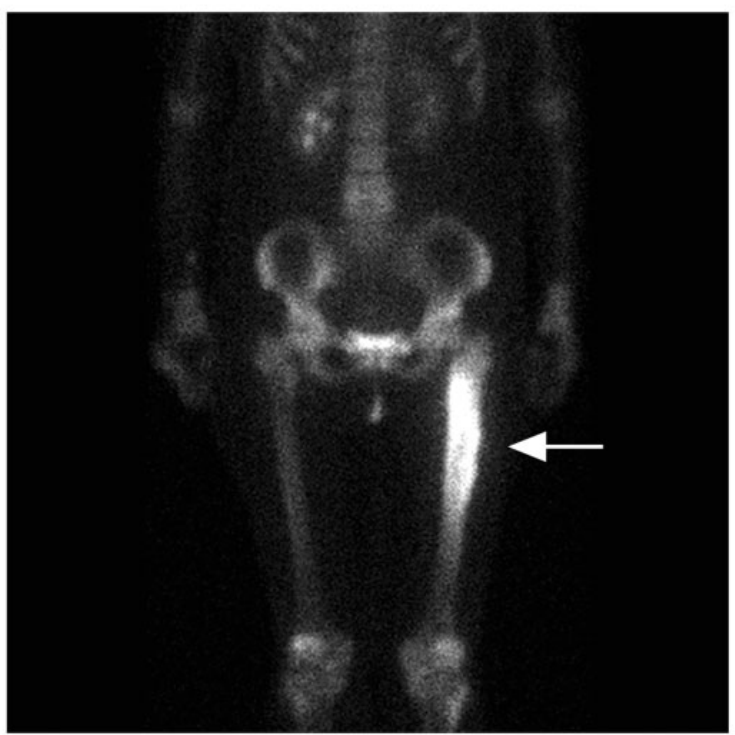

A lytic destructive appearance with a moth-eaten/ permeative wide zone of transition is the most common appearance (Fig. 1). Differential diagnosis of this appearance is varied and includes infection, eosinophilic granuloma, metastasis and Ewing sarcoma. Extra-osseous soft tissue mass and cortical breach leading to pathological fracture and periosteal reaction may be present, signifying advanced local disease (Fig. 2). The lytic lesions may also appear well defined, mimicking lesions of multiple myeloma. Periosteal reaction appears either as lamellated onion peel or discontinuous and are indicators of poor

(b)



(d)

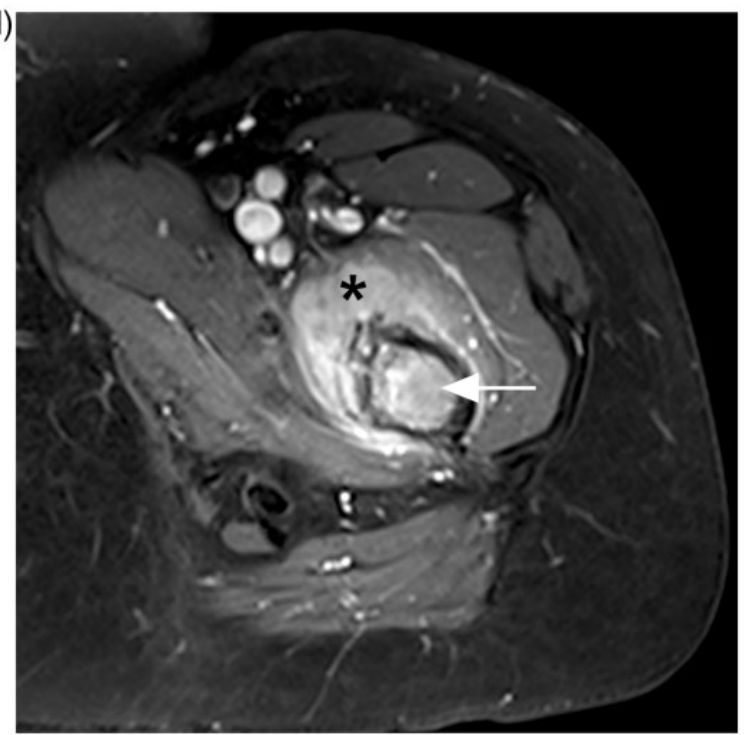

Figure 1 (a) Permeative lytic lesion in the proximal femur diaphysis (arrows) on the lateral left hip radiograph of a patient presenting with left hip pain. There is also a suggestion of irregular periosteal reaction (arrowhead). (b) Dedicated femur radiograph better shows a laminated peristeal reaction (arrowheads) but the permeative lytic lesion is more subtle (arrow). (c) Bone scan with technetium-99m methylene diphosphonate shows intense tracer uptake at the corresponding area. (d) Contrast-enhanced T1-weighted fat-saturated MR image showing marrow enhancement in the femur (arrow) and surrounding enhancing extra-osseous soft tissue mass (black asterisk). Biopsy subsequently confirmed a diagnosis of PBL. 
(a)

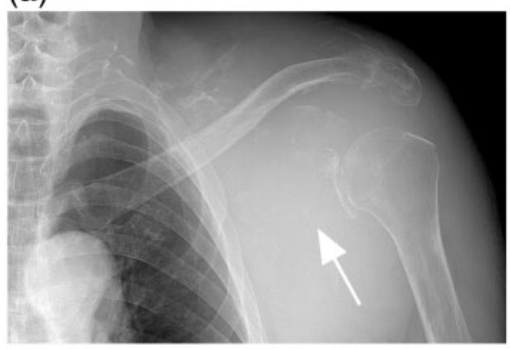

(d)



(b)



(e)

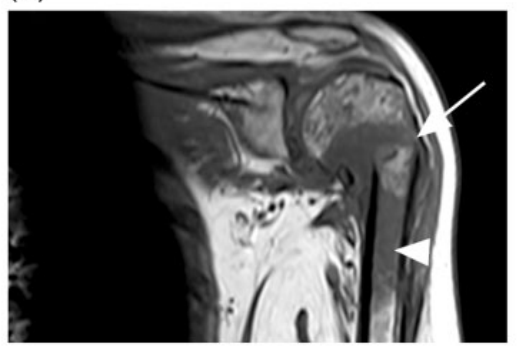

(c)

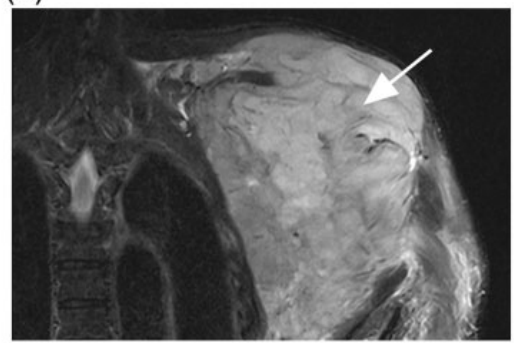

(f)

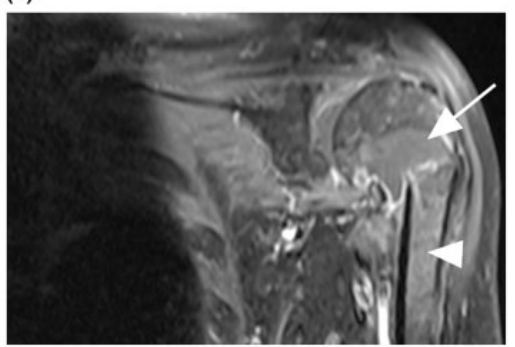

Figure 2 (a) Lytic destruction of the left scapula (white arrow) with soft tissue swelling in a patient with disseminated diffuse large B-cell lymphoma (DLBCL). (b) CT and (c) T2-weighted fat-saturated MR image of same patient showing the tumour mass (arrow). Associated axillary lymphadenopathy is also present (white arrowhead). (d) Another patient with disseminated DLBCL presented with pathological fracture of the left humeral neck. (e) T1-weighted and (f) contrast-enhanced T1-weighted fat-saturated MR images of the same patient showing a T1 hypointense tumour mass at the fracture site, which enhances after contrast (arrow). Marrow involvement of the humeral shaft is also present (arrowhead).

prognosis (Figs. 1, 3) ${ }^{[16]}$. Bone sequestrum is sometimes seen and the differential diagnosis includes osteomyelitis, bony tuberculosis, radiation necrosis and eosinophilic granuloma (Fig. 3) ${ }^{[7,18]}$.

A blastic-sclerotic pattern can appear as mixed lytic/ sclerotic lesions or, rarely, purely sclerotic lesions (Fig. 4). These lesions are described with Hodgkin disease, although most Hodgkin disease still shows a lytic radiological appearance ${ }^{[9]}$. Lytic lesions may also appear sclerotic after radiotherapy or chemotherapy ${ }^{[16]}$. A vertebral body showing diffuse sclerosis, or ivory vertebra, is a radiological sign associated with osseous lymphoma (Fig. 4); differentials include osteosarcoma, osteoblastic metastasis and Paget disease ${ }^{[19]}$.

Osseous lymphoma can also show subtle or normal appearance on radiographs but a florid appearance on other imaging modalities such as bone scintigraphy or magnetic resonance imaging (MRI) (Figs. 1, 5, 6). This is due to marrow infiltration and, although commonly described for osseous lymphoma, it is also found in other small round cell neoplasms such as Ewing sarcoma and multiple myeloma ${ }^{[17]}$. Because of this, further imaging for persistently symptomatic patients with normal radiographs is necessary to uncover underlying marrow disease.

Findings on computed tomography (CT) are similar to radiographic features described, but with better visualization of cortical and trabecular destruction, periosteal reaction, sequestra and extra-osseous masses (Figs. 2, 3).
Contrast-enhanced CT can also be used for studying marrow disease for planning bone biopsy in patients when MRI is contraindicated ${ }^{[20]}$.

MRI plays an integral role in the assessment of osseous lymphoma, showing high sensitivity for marrow replacement. Marrow involvement demonstrates low intensity on T1-weighted sequences and high intensity on T2weighted sequences; although if there is fibrosis within the tumour, low intensity on T2-weighted sequences may also be occasionally encountered. Short-tau inversion recovery (STIR) sequences are also helpful in the detection of abnormal marrow, whereas tumours typically show areas of enhancement after intravenous contrast administration (Figs. 2-6) ${ }^{[16]}$.

MRI is also useful for delineating associated extra-osseous soft tissue. The presence of a lytic permeative appearance of bone on radiographs with extensive marrow involvement and surrounding soft tissue mass is classic for round cell tumours such as lymphoma (Fig. 1) ${ }^{[16]}$. It is postulated that the tumour cells from the marrow invade small vascular channels within bone cortex into the surrounding soft tissue resulting in this appearance $^{[21]}$.

\section{Muscular disease}

Muscular lymphoma is rare, representing up to $1.4 \%$ of all malignant lymphomas ${ }^{[22]}$. It may occur as part of 


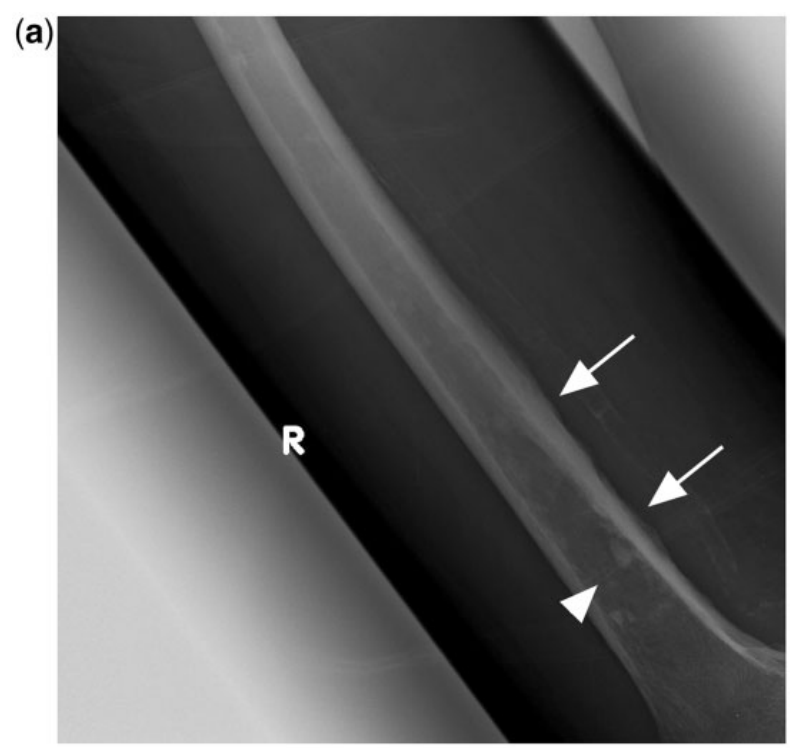

(b)
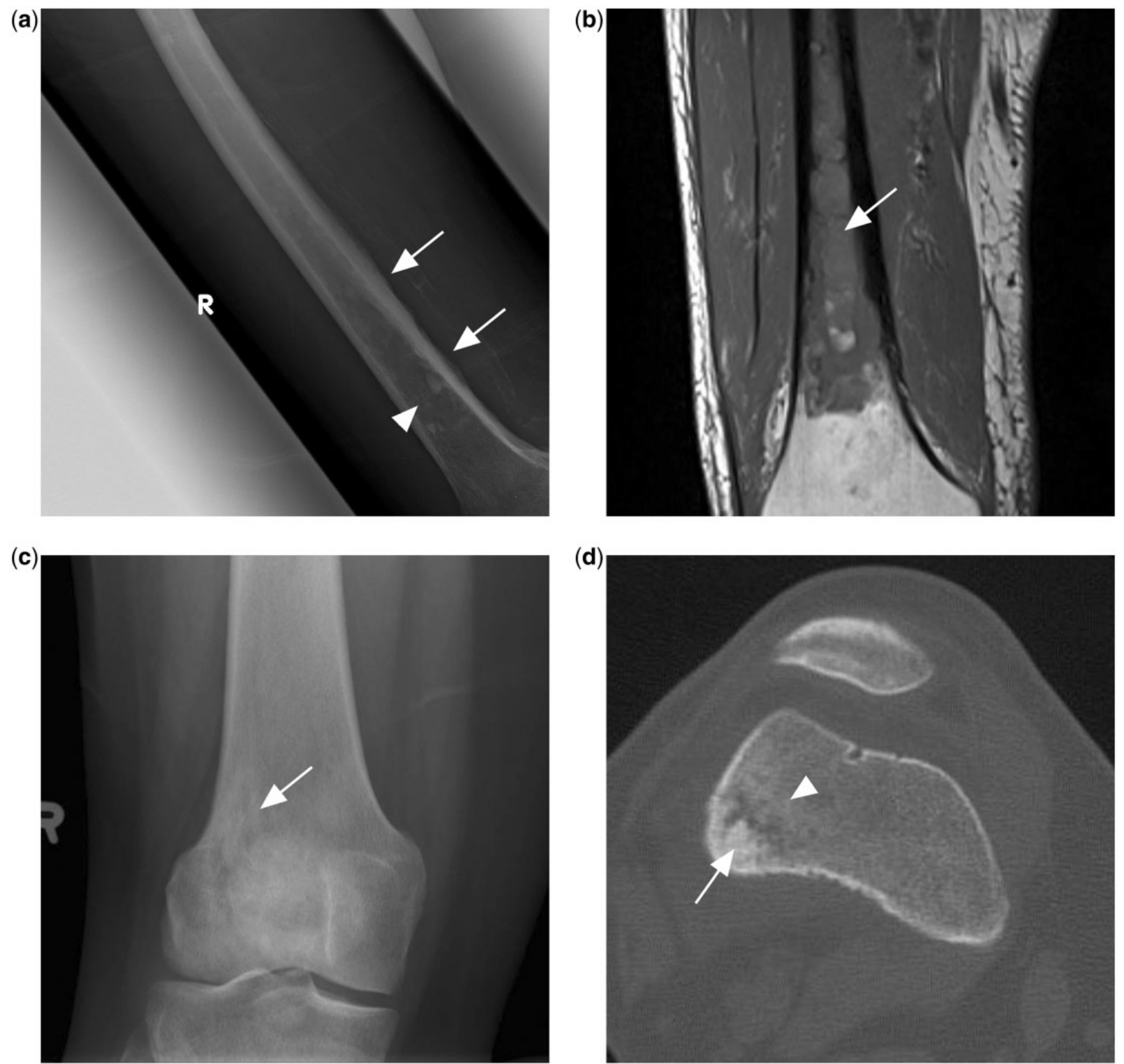

Figure 3 (a) Ill-defined mixed lytic sclerotic lesion in the mid to distal femoral shaft (arrowhead) with adjacent irregular periosteal reaction (arrows). (b) T1-weighted MR image of the same patient showing extensive T1 hypointense marrow changes in the femoral shaft, subsequently found to represent PBL. (c) Knee radiograph of another patient with PBL showing an ill-defined lytic sclerotic lesion (arrow) in the metaphysis of the distal femur. (d) CT scan of the same patient in the bone window shows central sclerotic sequestrum (arrow) with surrounding patchy sclerosis (arrowhead).

disseminated lymphoma, local extension from bone and lymphadenopathy or rarely primary muscular lymphoma $(\mathrm{PML})^{[23]}$. PML is exceedingly rare, seen in only 8 of 7000 patients with malignant lymphoma in a 10 -year study $^{[24]}$.

Muscular lymphoma is commonly due to NHL, including B-cell, T-cell, and natural killer cell types ${ }^{[22,25]}$. Clinical presentation may be acute or insidious, due to pain, swelling or B-symptoms ${ }^{[26]}$. PML occurs at a median age of 69 years ${ }^{[27]}$.

Muscular lymphoma is most common in the thigh and upper arm muscles ${ }^{[22,26,27]}$. It may present as a focal mass or diffuse muscular infiltration ${ }^{[28]}$. Ultrasonography features are non-specific and it appear as a heterogeneous, hypoechoic solid mass with irregular or poorly defined margins ${ }^{[26]}$. CT is also non-specific with tumour attenuation similar to or less than normal muscle and variable postcontrast enhancement (Fig. 7) $^{[26,28]}$.

MRI is the most useful modality for assessment of muscular lymphoma. The involved muscle shows enlargement, either with a discrete muscular mass or abnormal muscular signal intensity. The tumour is isointense or slightly hyperintense to normal muscle on 



(c)
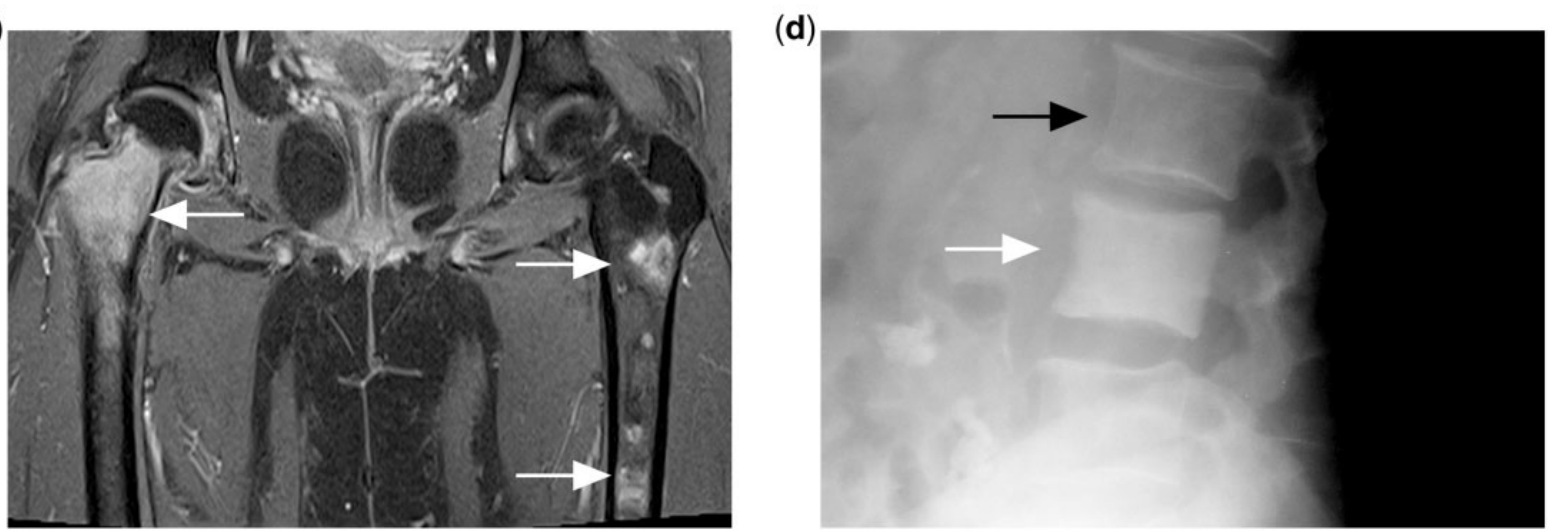

Figure 4 (a) Pelvic radiograph of a patient with disseminated Hodgkin lymphoma showing faint increased sclerosis in the right femur intertrochanteric region (white arrowhead) with layered periosteal reaction in the right proximal femur metadiaphyseal region (white arrow). Note apparent normal appearance of the left proximal femur. (b) T1-weighted and (c) contrast-enhanced T1-weighted fat-saturated MR images showing multiple foci of T1 hypointense and enhancing marrow lesions (white arrows) in both femora. (d) Diffusely increased sclerosis of the vertebral body (white arrow) compared with adjacent normal vertebral body (black arrow) is described as ivory vertebrae in another patient with Hodgkin lymphoma.

(a)

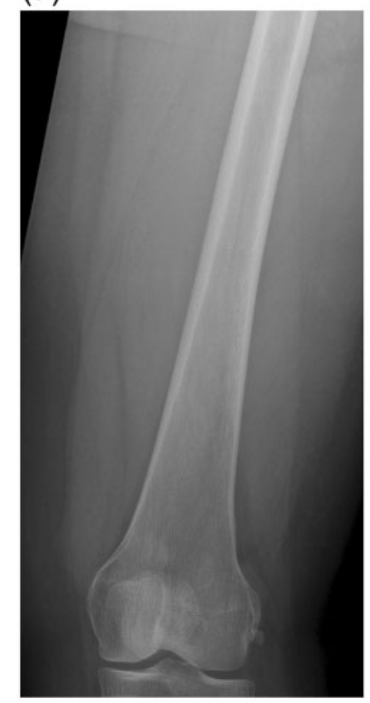

(b)

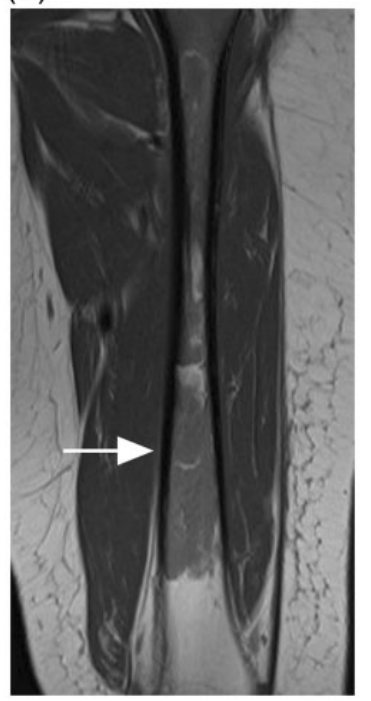

(c)

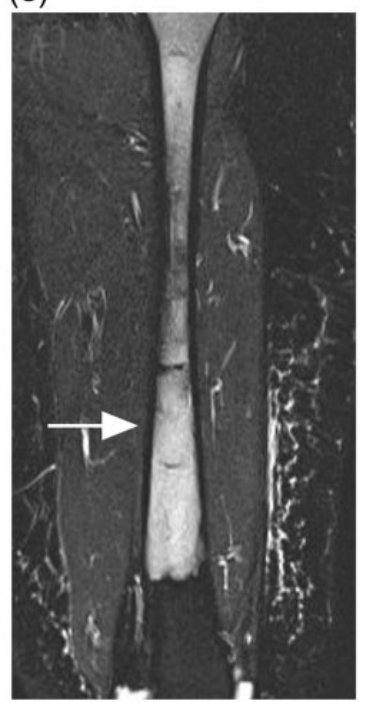

(d)

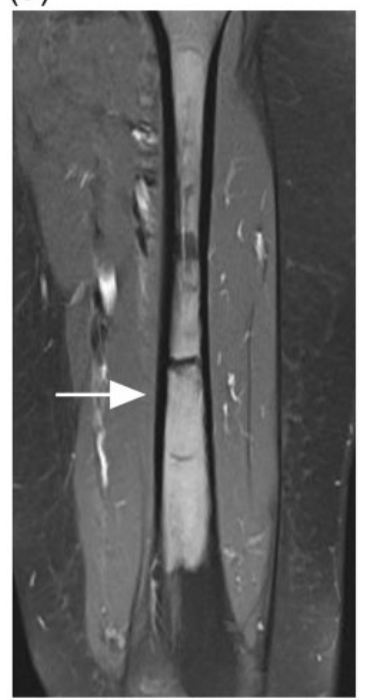

Figure 5 (a) Normal appearing radiograph of the femur of a patient who presented with persistent thigh pain. (b) T1weighted, (c) T2-weighted fat-saturated and (d) contrast-enhanced T1-weighted fat-saturated MR images reveal florid marrow disease that is T1 hypointense, T2 hyperintense and shows enhancement after contrast. Subsequent biopsy confirmed the diagnosis of PBL. 
(a)

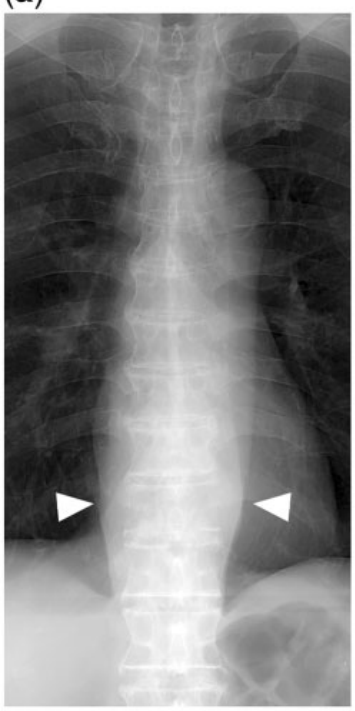

(b)

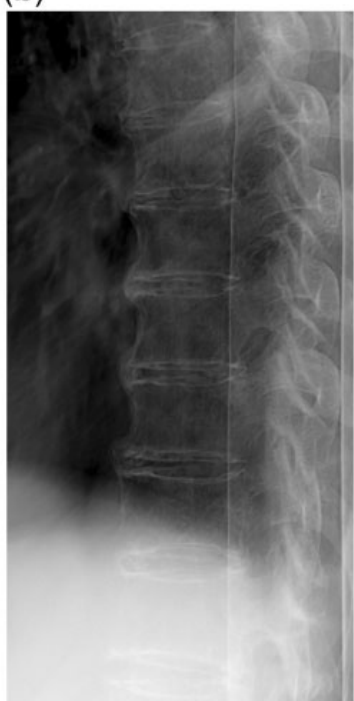

(c)

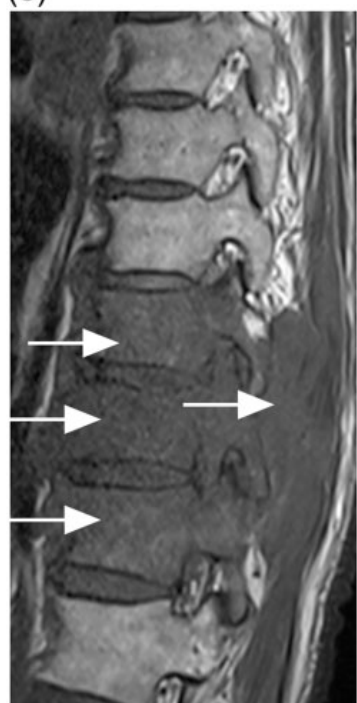

(d)

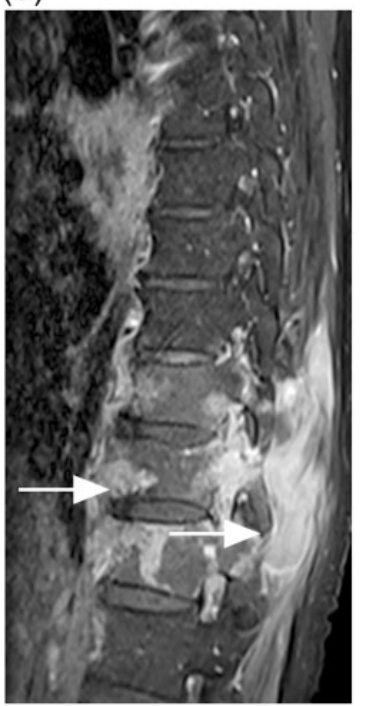

Figure 6 (a) Frontal and (b) lateral radiograph of the thoracic spine shows no obvious fracture or lytic destruction. However, widened paravertebral stripes are present (arrowheads). (c) T1-weighted and (d) contrast-enhanced T1weighted fat-saturated MR images show extensive enhancing hypointense marrow disease with extra-osseous soft tissue (arrows) in the lower thoracic spine secondary to disseminated DLBCL.

(a)

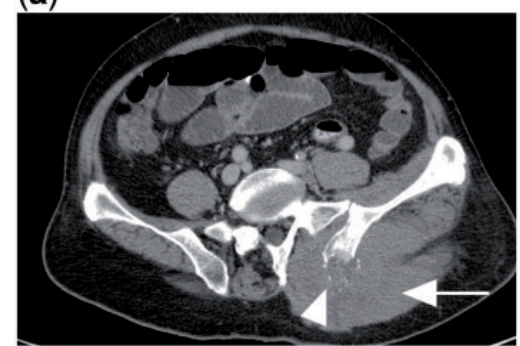

(d)

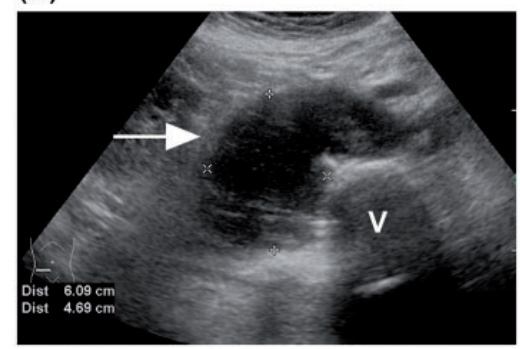

(b)

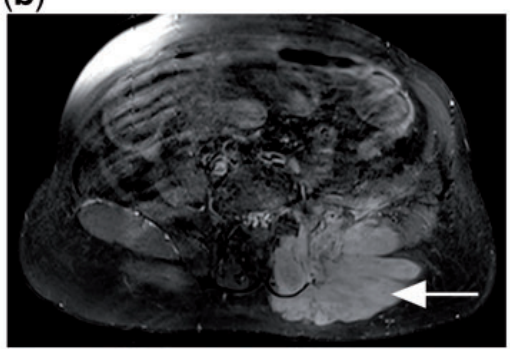

(e)

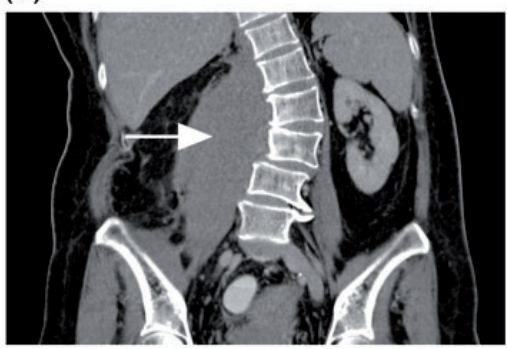

(c)

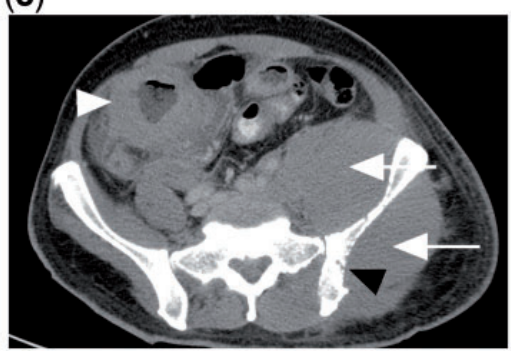

(f)

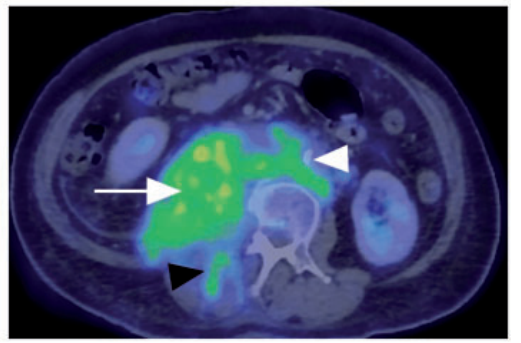

Figure 7 (a) CT of a patient with disseminated DLBCL showing diffuse enlargement of the left gluteus muscle (white arrow) with adjacent lytic destruction of the left iliac bone (white arrowhead). (b) Contrast-enhanced T1-weighted fatsaturated MR image of the same patient showing diffusely enlarged and enhancing left gluteus and erector spinae muscles. (c) CT of a different patient with Burkitt lymphoma of the colon (white arrowhead). There is diffuse involvement of the left gluteus and iliacus muscles (white arrows) with erosion of underlying iliac bone (black arrowhead). (d) Ultrasonograph of the abdomen of another patient with abdominal pain shows a hypoechoic right paravertebral mass. $\mathrm{V}$ indicates vertebral body. (e) Coronal CT of the same patient shows the ultrasonographic finding to be a diffusely enlarged right psoas muscle, subsequently diagnosed as marginal zone lymphoma on biopsy. (f) PET/CT with FDG showing avidity of the tumour (white arrow), which extends to the right erector spinae muscle (black arrowhead) as well as retroperitoneal lymphadenopathy (white arrowhead). 
T1-weighted sequences and of intermediate signal intensity between muscle and fat on T2-weighted sequences. Tumour commonly enhances after contrast, either as diffuse homogeneous, predominately peripheral thick band-like enhancement or marginal septal enhancement. Thick irregular enhancement of both deep and/or superficial fascia can also occur. Features occasionally seen but suggestive of lymphoma include adjacent subcutaneous stranding with or without skin thickening, long segmental involvement with orientation of the tumour along muscle fascicles, traversing vessels within involved muscles, involvement of more than one muscle compartment and extension of tumour along the neurovascular bundle (Fig. 8) ${ }^{[25,29]}$.

\section{Skin disease}

Lymphoma of the skin can occur primarily or secondarily as part of disseminated disease. Primary cutaneous lymphoma refers to cutaneous T-cell lymphomas (CTCL) and cutaneous B-cell lymphomas (CBCL) in the skin with no evidence of extracutaneous disease at the time of diagnosis. This is the second most common site of extranodal NHL after the gastrointestinal system, with an estimated annual incidence of 1 in $100,000^{[30]}$.

The most common form, mycosis fungoides (CTCL), occurs at a median age of 55-60 years at diagnosis and shows a male preponderance. The clinical course is indolent with slow progression over years from patches to infiltrative plaques and subsequently tumours that often (a)

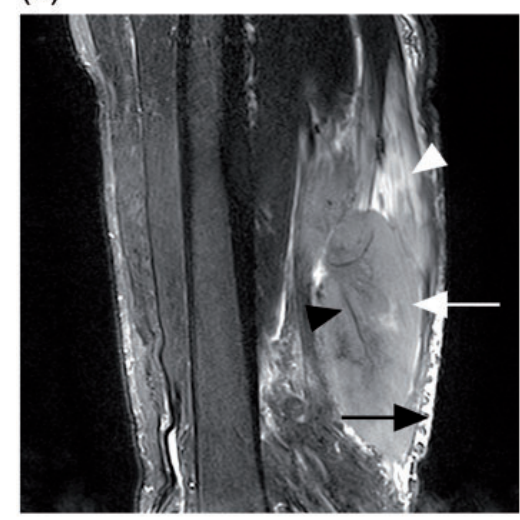

(d)

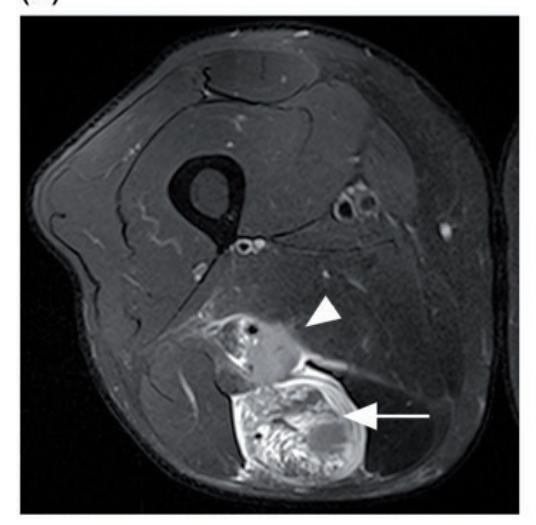

(b)

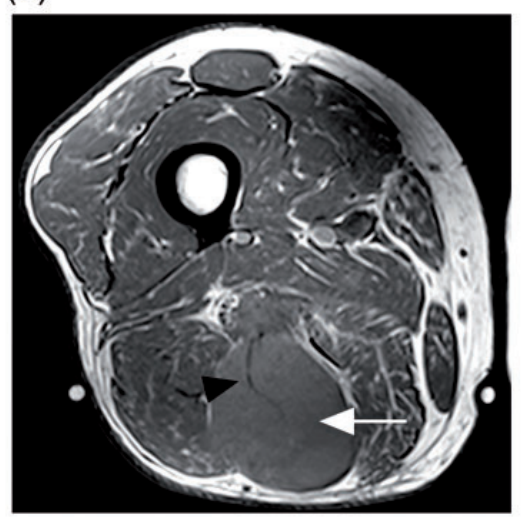

(e)

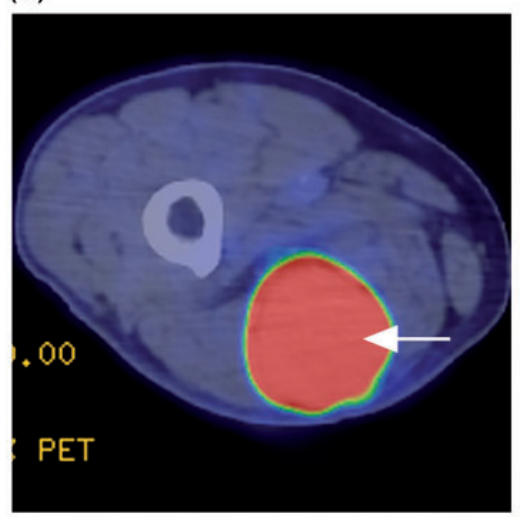

(c)

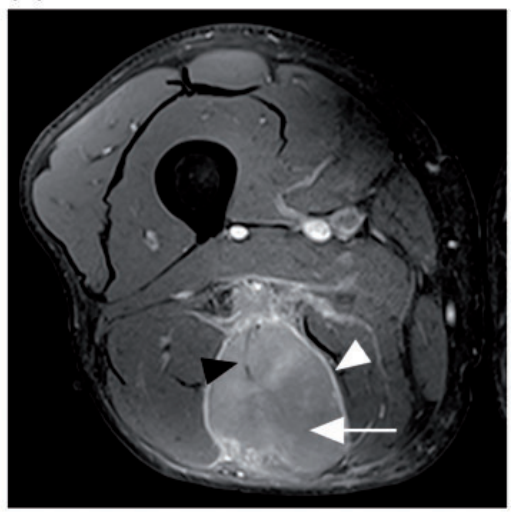

(f)

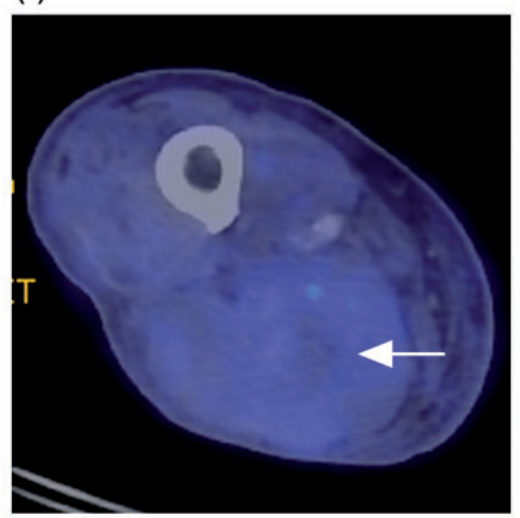

Figure 8 Patient with a primary lymphoma in the semitendinosus muscle. (a) Sagittal T2-weighted fat-saturated MR image showing a T2 hyperintense tumour mass within the muscle (white arrow), long segmental involvement with orientation of the tumour along muscle fascicles (white arrowhead), traversing vessels within involved muscle (black arrowhead) and adjacent subcutaneous stranding (black arrow). (b) Axial T1 MR image showing a tumour of slightly increased signal intensity (white arrow) compared with normal muscles and a traversing vessel (black arrowhead). (c) Contrast-enhanced T1-weighted fat-saturated MR image (white arrow) with peripheral septal enhancement (white arrowhead). Traversing vessel noted again (black arrowhead). (d) Axial T2-weighted fat-saturated MR image showing a heterogeneous T2 hyperintense tumour (white arrow) and adjacent neurovascular bundle involvement (white arrowhead). (e) Prechemotherapy PET/CT with FDG shows the tumour to be FDG avid (white arrow). (f) Postchemotherapy PET/ CT showing decrease in FDG avidity of the tumour (white arrow), compatible with treatment response. 
(a)

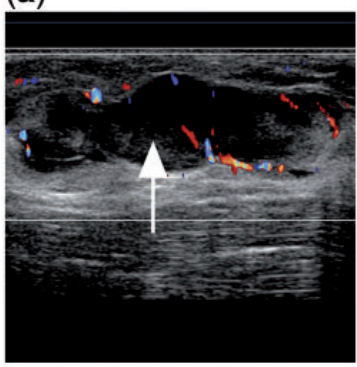

(b)

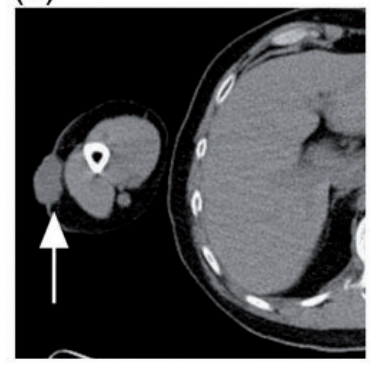

(c)

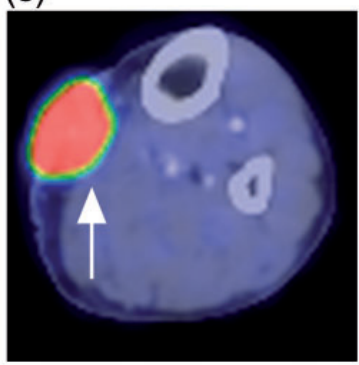

(d)

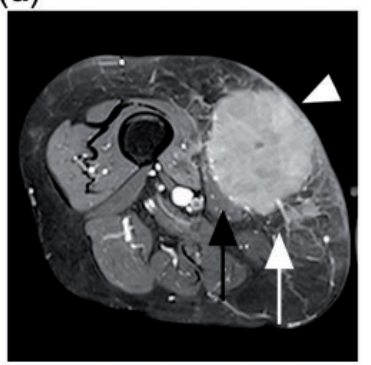

Figure 9 (a) Ultrasonograph of a patient with mycosis fungoides showing a dermal lobulated hypoechoic mass with internal vascularity (white arrow). (b) CT of the same patient demonstrates the cutaneous soft tissue mass in the right arm (white arrow). (c) PET/CT with FDG of another patient with mycosis fungoides in the left calf showing an FDG-avid cutaneous soft tissue mass (white arrow). (d) Contrast-enhanced T1-weighted fat-saturated MR image of a different patient with disseminated DLBCL showing an enhancing subcutaneous tumour mass in the medial thigh (white arrow) invading into the skin (white arrowhead) and adjacent sartorius muscle (black arrow).

(a)

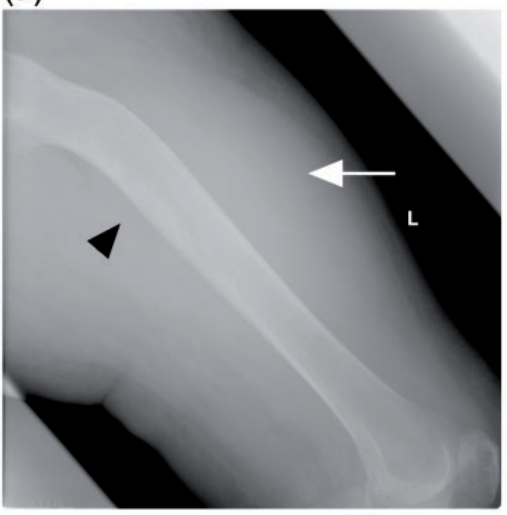

(d)

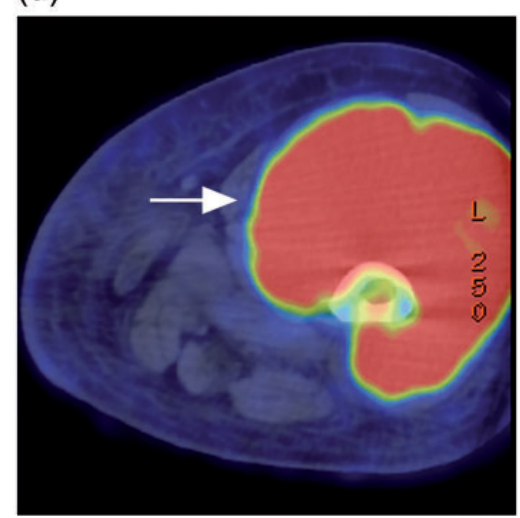

(b)

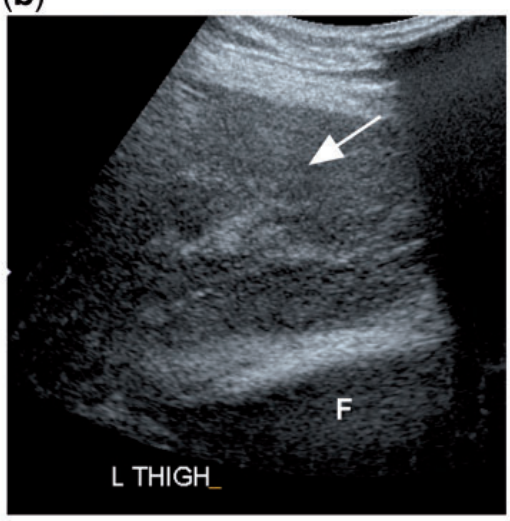

(e)

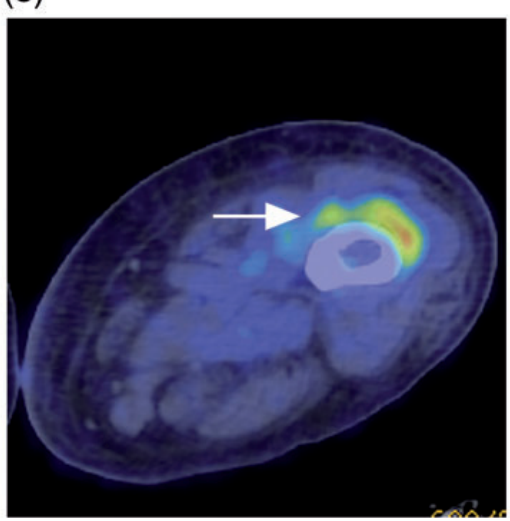

(c)

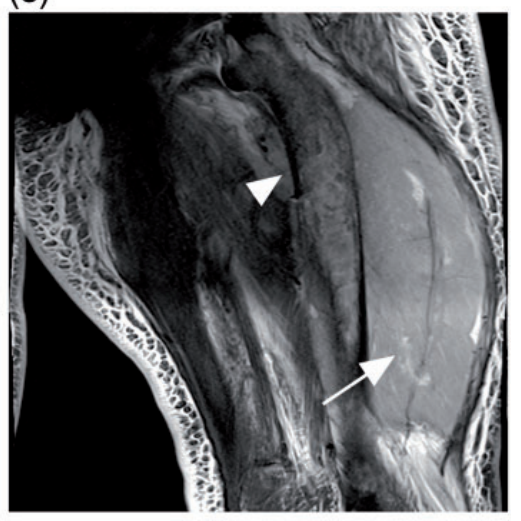

(f)

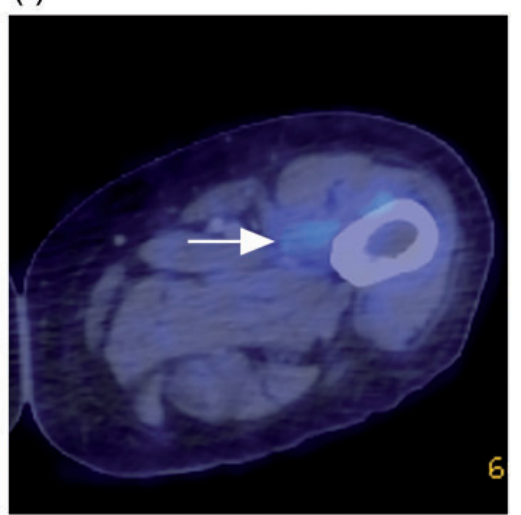

Figure 10 A patient with disseminated follicular lymphoma presented with left thigh swelling. (a) Radiograph of the left femur showing soft tissue swelling in the left thigh (white arrow) with sclerosis and bowing of the left femoral shaft (black arrowhead). (b) Ultrasonograph of the left thigh showing a large heterogeneous swelling of the left quadriceps muscle. F indicates femur. (c) T2-weighted fat-saturated image showing swelling with diffusely raised signal intensity of the quadriceps muscle (white arrow). Marrow involvement of the underlying femur (white arrowhead) also noted. (d) Prechemotherapy PET/CT with FDG showing FDG avidity of the tumour mass. (e, f) Interval PET/CT after chemotherapy showing progressive decrease in size and FDG avidity of the tumour compatible with treatment response (white arrow). 
ulcerate. Lymph nodes and visceral organ involvement signifies late disease stage ${ }^{[31]}$.

Imaging is non-specific and plays a limited role in diagnosis, which still depends on histology. Depending on the stage of disease, cutaneous lymphoma may appear as diffuse thickening or a focal mass/nodule. Ultrasonography shows a well-defined hypoechoic nodule, which may coalesce into a polypoidal patch in the focal type, and homogeneous hyperechoic dermal thickening or inhomogeneous dermal and subcutaneous thickening in the diffuse type ${ }^{[32]}$. CT shows either diffuse skin thickening or focal skin nodule/mass and is more useful as a staging tool to detect lymphadenopathy or visceral disease involvement $^{[28,33]}$. MRI features are equally non-specific but useful for assessment of local disease extent (Fig. 9) ${ }^{[34,35]}$.

\section{Post-treatment imaging}

Assessment of post-treatment response via imaging is largely achieved using positron emission tomography (PET)/CT with $\left[{ }^{18} \mathrm{~F}\right]$ fluorodeoxyglucose (FDG) and $\mathrm{MRI}^{[36,37]}$. Change in size and FDG avidity are now the main imaging parameters assessed under the revised International Working Group Response Criteria ${ }^{[38]}$. PET has an advantage over CT/MRI because of its ability to distinguish between viable tumour and necrosis/fibrosis in residual masses after treatment, provided the pretreatment scan showed the tumour to be FDG avid (Figs. 8, 10). Assessment of early response after initiation of chemotherapy through PET/CT can be used to predict the final treatment response and progression-free survival, as well as potentially identify patients requiring more aggressive treatment ${ }^{[37]}$. Most subjective indicators of treatment response on MRI include decrease in tumour signal intensity on T2-weighted sequences and decrease in contrast enhancement.

\section{Conclusion}

Lymphoma is not uncommon and musculoskeletal manifestations may be the presenting symptom. A definitive diagnosis is difficult to make with imaging because of non-specific features and considerable overlap with other pathologies. A high index of suspicion, recognition of characteristic features across various imaging modalities, as well as knowledge of the clinical features will help suggest the differential diagnosis and direct the clinical investigation. Imaging also plays an integral role in treatment response assessment and potentially influences management.

\section{Conflict of interest}

The authors have no conflicts of interest to declare.

\section{References}

[1] Campo E, Swerdlow SH, Harris NL, Pileri S, Stein H, Jaffe ES. The 2008 WHO classification of lymphoid neoplasms and beyond: evolving concepts and practical applications. Blood 2011; 117: 5019-5032.

[2] Kwee TC, Kwee RM, Nievelstein RA. Imaging in staging of malignant lymphoma: a systematic review. Blood 2008; 15: 504-516.

[3] Ministry of Health Singapore, National Cancer Registry Interim Annual Registry Report Trends in Cancer Incidence in Singapore 2007-2011. http://www.nrdo.gov.sg/uploadedFiles/NRDO/Can cer_Trends_Report_07-11_for_website_v2.pdf. Accessed 24th February 2013.

[4] Jemal A, Siegel R, Ward E, Murray T, Xu J, Thun MJ. Cancer statistics, 2007. CA Cancer J Clin 2007; 57: 43-66.

[5] Ostrowski ML, Unni KK, Banks PM, et al. Malignant Lymphoma of bone. Cancer 1986; 58: 2646-2655.

[6] Suresh S, Saifuddin A, O’Donnell P. Lymphoma presenting as a musculoskeletal soft tissue mass: MRI finding in 24 cases. Eur Radiol 2008; 18: 2628-2634.

[7] Fletcher C, Unni K, Mertens F. Pathology and genetics of tumours of soft tissue and bone: World Health Organization classification of tumours. Lyon, France: International Agency for Research on Cancer; 2002, p. 306-308.

[8] Coley BL, Higinbotham NL, Groesbeck HP. Primary reticulum cell sarcoma of the bone; summary of 37 cases. Radiology 1950; 55: 641-658.

[9] O’Neill J, Finlay K, Friedman L. Radiological manifestations of skeletal lymphoma. Curr Probl Diagn Radiol 2009; 38: 228-236.

[10] Beal K, Allen L, Yahalom J. Primary bone lymphoma: treatment results and prognostic factors with long term follow-up of 82 patients. Cancer 2006; 106: 2652-2656.

[11] Pires de Camargo O, Machado TMS, Croci AT, et al. Primary bone lymphoma in 24 patients treated between 1955 and 1999. Clin Orthop 2002; 397: 271-280.

[12] Ebus SC, Bernsen HJ, Norel GJ, Donk R. Primary non-Hodgkin's lymphoma in multiple vertebrae presenting as a radicular syndrome. Spine 2002; 27: E271-E273.

[13] Mulligan ME, McRae GA, Murphey MD. Imaging features of primary lymphoma of bone. AJR Am J Roentgenol 1999; 173: 1691-1697.

[14] Abdelwahab IF, Miller TT, Hermann G, Klein MJ, Kenan S, Lewis MM. Transarticular invasion of joints by bone tumors: hypothesis. Skeletal Radiol 1991; 20: 279-283.

[15] Melamed JW, Martinez S, Hoffman CJ. Imaging of primary multifocal osseous lymphoma. Skeletal Radiol 1997; 26: 35-41.

[16] Krishnan A, Shirkhoda A, Tehranzadeh J, Armin AR, Irwin R, Les K. Primary bone lymphoma: radiographic-MR imaging correlation. Radiographics 2003; 23: 1371-1387.

[17] Mulligan ME, Kransdorf MJ. Sequestra in primary lymphoma of bone. AJR Am J Roentgenol 1993; 160: 1245-1248.

[18] Jennin F, Bousson V, Parlier C, Jomaah N, Khanine V, Laredo JD. Bony sequestrum: a radiologic review. Skeletal Radiol 2011; 40: 963-975.

[19] Graham TS. The ivory vertebra sign. Radiology 2005; 235 : 614-615.

[20] Hwang S. Imaging of lymphoma of the musculoskeletal system. Radiol Clin North Am 2008; 46: 379-396.

[21] Hicks DG, Gokan T, O'Keefe RJ, et al. Primary lymphoma of bone: correlation of magnetic resonance imaging features with cytokine production by tumor cells. Cancer 1995; 75: 973-980.

[22] Komatsuda M, Nagao T, Arimori S. An autopsy case of malignant lymphoma associated with remarkable infiltration in skeletal muscles. Rinsho Ketsueki 1981; 22: 891-895.

[23] Lee VS, Martinez S, Coleman RE. Primary muscle lymphoma: clinical and imaging findings. Radiology 1997; 203: 237-244.

[24] Travis WD, Banks PM, Reiman HM. Primary extranodal soft tissue lymphoma of the extremities. Am J Surg Pathol 1987; 11: $359-366$. 
[25] Chun CW, Jee WH, Park HJ, et al. MRI features of skeletal muscle lymphoma. AJR Am J Roentgenol 2010; 195: 1355-1360.

[26] Beggs I. Primary muscle lymphoma. Clin Radiol 1997; 52: 203-312.

[27] Samuel LM, White J, Lessells AM, Roddie H, Matheson LM. Primary non-Hodgkins lymphoma of muscle. Clin Oncol 1999; 11: 49-51.

[28] Malloy PC, Fishman EK, Magid D. Lymphoma of bone, muscle and skin: CT findings. AJR Am J Roentgenol 1992; 159: 805-809.

[29] Suresh S, Saifuddin A, O'Donnell P. Lymphoma presenting as a musculoskeletal soft tissue mass: MRI findings in 24 cases. Eur Radiol 2008; 18: 2628-2634.

[30] Groves FD, Linet MS, Travis LB, Devesa SS. Cancer surveillance series: non-Hodgkin's lymphoma incidence by histologic subtype in the United States from 1978 through 1995. J Natl Cancer Inst 2000; 92: 1240-1251.

[31] Willemze R, Jaffe ES, Burg G, et al. WHO-EORTC classification for cutaneous lymphomas. Blood 2005; 105: 3768-3785.
[32] Giovagnorio F. Sonography of cutaneous non-Hodgkin's lymphomas. Clin Radiol 1997; 52: 301-303.

[33] Lee HJ, Im JG, Goo JM, et al. Peripheral T-cell lymphoma: spectrum of imaging findings with clinical and pathologic features. Radiographics 2003; 23: 7-26.

[34] Vanhoenacker FM, Baten A, Vandeputte V. Imaging findings of a cutaneous B-cell lymphoma. JBR-BTR 2009; 92: 285-288.

[35] Beaman FD, Kransdorf MJ, Andrews TR, Murphey MD, Arcara LK, Keeling JH. Superficial soft-tissue masses: analysis, diagnosis, and differential considerations. Radiographics 2007; 27: 509-523.

[36] Rahmouni A, Luciani A, Itti E. MRI and PET in monitoring response in lymphoma. Cancer Imaging 2005; 5(Spec No A): S106-S112.

[37] Hutchings M, Barrington SF. PET/CT for therapy response assessment in lymphoma. J Nucl Med 2009; 50(Suppl 1): $21 \mathrm{~S}-30 \mathrm{~S}$.

[38] Cheson BD, Pfistner B, Juweid ME, et al. Revised response criteria for malignant lymphoma. J Clin Oncol 2007; 25: 579-586. 